Check for updates

Cite this: Phys. Chem. Chem. Phys., 2018, 20, 16579

Received 6th March 2018, Accepted 14th May 2018

DOI: $10.1039 / c 8 c p 01485$

rsc.li/pccp

\section{Understanding transport mechanisms in ionic liquid/carbonate solvent electrolyte blends $\dagger$}

\author{
K. Oldiges, ${ }^{a b}$ D. Diddens, (D) a M. Ebrahiminia, ${ }^{c}$ J. B. Hooper, ${ }^{c}$ I. Cekic-Laskovic, ${ }^{\text {abd }}$ \\ A. Heuer, (D) ${ }^{a b}$ D. Bedrov, (D) $* c$ M. Winter ${ }^{a b d}$ and G. Brunklaus (iD *abd
}

\begin{abstract}
To unravel mechanistic details of the ion transport in liquid electrolytes, blends of the ionic liquid (IL) 1-butyl-1-methylpyrrolidinium bis(trifluoromethylsulfonyl)imide ( $\mathrm{Pyr}_{14} \mathrm{TFSI}$ ), ethylene carbonate (EC) and dimethyl carbonate (DMC) with the conducting salts lithium hexafluorophosphate $\left(\mathrm{LiPF}_{6}\right)$ and lithium bis(trifluoromethylsulfonyl)imide (LiTFSI) were investigated as a function of the IL concentration. Electrochemical impedance, Pulsed Field Gradient Nuclear Magnetic Resonance (PFG NMR) and Raman spectroscopy supported by Molecular Dynamics (MD) simulations allowed the structural and dynamic correlations of the ion motions to be probed. Remarkably, we identified that though the individual correlations among different ion types exhibit a clear concentration dependence, their net effect is nearly constant throughout the entire concentration range, resulting in approximately equal transport and transference numbers, despite a monitored cross-over from carbonate-based lithium coordination to a TFSI-based ion coordination. In addition, though dynamical ion correlation could be found, the absolute values of the ionic conductivity are essentially determined by the overall viscosity of the electrolyte. The IL/carbonate blends with a $\mathrm{Pyr}_{14} \mathrm{TFSI}$ fraction of $\sim 10 \mathrm{wt} \%$ are found to be promising electrolyte solvents, with ionic conductivities and lithium ion transference numbers comparable to those of standard carbonate-based electrolytes while the thermal and electrochemical stabilities are considerably improved. In contrast, the choice of the conducting salt only marginally affects the transport properties.
\end{abstract}

\section{Introduction}

Contemporary lithium-ion battery technology facilitates a broad variety of applications including portable electronics, electric transport or grid solutions. Despite considerable efforts and recent advances in the tailored design of electrolyte components (lithium salts, solvents or additives), substantial challenges such as stabilization of electrolyte/cathode interfaces and interphases at higher potentials $\left(>4.5 \mathrm{~V}\right.$ vs. $\left.\mathrm{Li} / \mathrm{Li}^{+}\right)$, better compatibility of electrolytes with metallic anodes or "beyond lithium-ion" chemistries (e.g., $\mathrm{Li}-\mathrm{S}$ and $\mathrm{Li}-\mathrm{O}_{2}$ batteries) and further development of sustainable materials and processes for battery manufacture remain to be solved..$^{1-7}$ Since liquid electrolytes, commonly used in current batteries, affect the achievable power output and hence electrochemical performance of the cells, it is crucial to

\footnotetext{
${ }^{a}$ Helmholtz Institute Münster, IEK-12, Forschungszentrum Jülich GmbH, Corrensstrasse 46, 48149 Münster, Germany.E-mail: g.brunklaus@fz-juelich.de

${ }^{b}$ Institute of Physical Chemistry, University of Münster, Corrensstrasse 28/30, 48149 Münster, Germany

${ }^{c}$ Department of Materials Science \& Engineering, University of Utah, 122 South Central Campus Drive, Salt Lake City, Utah 84112, USA. E-mail: d.bedrov@utah.edu

${ }^{d}$ MEET Battery Research Center, Corrensstrasse 46, 48149 Münster, Germany

$\dagger$ Electronic supplementary information (ESI) available. See DOI: 10.1039/c8cp01485j
}

better understand not only processes at electrolyte/electrode interfaces but also ion transport mechanisms within considered electrolytes that critically determine the mobility and availability of electroactive species for the occurring electrochemical reactions. Indeed, many different requirements have to be fulfilled by applicable electrolyte solvents or conducting salts, including sufficient solubility and degree of dissociation of salts in nonaqueous solvents as well as affording high ion mobility, while simultaneously being inert towards all cell components. Liquid electrolytes should possess low viscosity and high ionic conductivity in order to facilitate fast ion transport, and it was shown that modest improvements in transport numbers are beneficial to allow for higher attainable state of charge at higher rates. ${ }^{8,9}$ Abundantly applied state-of-the-art electrolytes are based on organic carbonate solvents and LiPF $_{6}$ and have limited operational safety due to their moisture sensitivity and at elevated temperature, flammability and volatility. ${ }^{2,10-12}$ Further improvement of electrolyte properties such as stability with respect to high energy electrodes (e.g., lithium metal), ionic conductivity and transport remain major challenges for future performance gains, including polymer electrolytes. ${ }^{13-16}$ Indeed, mixing of ionic liquids, solvents and appropriate conducting salts constitutes a particularly viable strategy for materials development and optimization. ${ }^{17,18}$ 
Among the available alternatives, room temperature ionic liquids (RTILs) offer superior thermal and electrochemical stabilities $^{3,19}$ but unfavorably high viscosity, which according to the Stokes-Einstein equation has negative impact on ion transport properties. ${ }^{19,20}$ Therefore, several efforts were made to improve ion mobility in ionic liquids thereby making them more attractive for lithium-ion battery applications. One approach involves mixing of ILs where the resulting blends offer more beneficial properties than individual single compounds, ${ }^{21}$ such as e.g. lower melting points or higher ionic conductivities even at low temperatures. Another strategy includes the addition of small organic compounds like EC, vinylene carbonate (VC), acetonitrile (AN) or tetrahydrofuran (THF) which based on molecular dynamics (MD) simulations afford lesser coordination of lithium ions with the anion of the ILs and hence better mobility and ionic conductivity. ${ }^{22,23}$ While blends of organic solvents are a commodity, ${ }^{24,25}$ blends of ILs and organic carbonates were considered by several groups only recently ${ }^{26-33}$ particularly focusing on improved safety performance, reduced aluminum current collector dissolution or enhanced electrochemical properties. While selected features of the ion transport in pristine ILs or ILs doped with lithium salts were previously described,,$^{20,34-37}$ the situation is less clear in case of ternary mixtures. Among the large variety of possible ILs, Pyr ${ }_{14}$ TFSI is particularly attractive due to its high decomposition temperature of $360{ }^{\circ} \mathrm{C}$ and its ability to improve both operational safety and ionic conductivity of carbonate-based electrolytes. Macroscopic properties such as thermal properties of $\mathrm{Pyr}_{14}$ TFSI/EC/DMC blends ${ }^{38}$ are commonly considered, while often neglecting the molecular details or ion speciation that govern the achievable ion transport properties of such blends, except for recent work. ${ }^{39}$ However, when aiming at a comprehensive understanding of probable ion transport processes in $\mathrm{Pyr}_{14}$ TFSI/EC/DMC blends in the presence of lithium salts, it is important to consider macroscopic properties of electrolytes including viscosities, self-diffusion coefficients, molar conductivities, apparent activation energies or transport numbers and the degree of ion dissociation, respectively. Note that the anion of the selected conducting salt typically corresponds to the anion of ILs. Despite the fact that $\mathrm{LiPF}_{6}$ is an industry standard and abundantly used in organic carbonate-based electrolytes, ${ }^{40,41}$ it was not yet considered in $\mathrm{IL} /$ carbonate blends, though electrolytes comprised of $1 \mathrm{M} \mathrm{LiPF}_{6}$ in equimolar mixtures of EC/DMC revealed better ionic conductivity $\left(10.7 \mathrm{mS} \mathrm{cm} \mathrm{cm}^{-1}\right)$ at room temperature compared to similar blends with LiTFSI $\left(9.4 \mathrm{mS} \mathrm{cm}^{-1}\right) .{ }^{42}$ Most ionic liquids studied for electrochemical applications are based on the anion TFSI $^{-}$, rendering LiTFSI a suitable option as long as its significant costs and deleterious aluminum dissolution are neglected. ${ }^{43-46}$ Therefore, it appears useful to critically compare the impact of both $\mathrm{LiPF}_{6}$ and LiTFSI on the achievable ion transport properties of the corresponding $\mathrm{IL} /$ carbonate blends.

In this work, we conducted a systematic experimental and computational study of bulk properties of IL/carbonate blends particularly unraveling the influence of both actual amounts of $\mathrm{Pyr}_{14}$ TFSI and the presence of a fourth ion species (i.e., $\mathrm{PF}_{6}{ }^{-}$) on the resulting ion transport processes or lithium speciation. Electrochemical impedance spectroscopy (EIS) and viscosity measurements were performed at variable temperatures while the self-diffusion coefficients of all species (which are the basis for calculation of important parameters) were determined from PFG NMR spectroscopy. Complementary insight concerning the ion speciation within the IL/carbonate blends was derived from Raman spectroscopy and in particular atomistic molecular dynamics simulations utilizing polarizable force fields thereby revealing microscopic details of the ion transport processes.

\section{Experimental}

\section{Sample preparation}

LiTFSI (3 M, Fluorad ${ }^{\mathrm{TM}}$ HQ-115) was dried under vacuum at $110{ }^{\circ} \mathrm{C}$, while $\mathrm{LiPF}_{6}$ (BASF, battery grade), EC (Merck, SelectiLyte ${ }^{\mathrm{TM}}$ ) and DMC (BASF, Selectilyte ${ }^{\mathrm{TM}}$ ) were used as received. Pyr $_{14}{ }^{\mathrm{TFSI}}$ (Solvionic) was dried with a turbo pump vacuum system at $110{ }^{\circ} \mathrm{C}$. All blends were prepared in an argon atmosphere (glove box) using $1 \mathrm{M}$ conducting salt in $x$ wt $\% \operatorname{Pyr}_{14}$ TFSI and $(100-x)$ wt $\%$ EC: DMC (1:1 by wt), respectively, with $x=0,10,30,40,50,70$, and 100.

\section{Impedance measurements}

EIS measurements were performed with a Metrohm potentiostat (Autolab PGSTAT302N ${ }^{\circledR}$ ) using the FRA32 module as frequency range scanner and the software program NOVA ${ }^{\circledR}$ 1.10.2 (Metrohm Autolab B.V.). Micro-electrode liquid measurement cells ${ }^{47,48}$ were used in a 16-cell arrangement (ESI $\dagger$ ), where the actual cell temperature was monitored by a Pt1000 element immersed in EC/DMC (1:1 by wt) solution (located within a 17th sample container). The impedance was measured in the temperature range from -10 to $40{ }^{\circ} \mathrm{C}$, in steps of $5{ }^{\circ} \mathrm{C}$. Fifty measuring points were determined in a frequency range from $10 \mathrm{~Hz}$ to $50000 \mathrm{~Hz}$ with an AC voltage of $40 \mathrm{mV}$; each measurement was repeated three times for data reproducibility.

\section{PFG NMR measurements}

Self-diffusion coefficients of all electrolyte species were derived by PFG NMR. All measurements were performed with stimulated echo sequences at a Bruker AVANCE III 200 spectrometer using a Bruker Diff30 probe at $25.0{ }^{\circ} \mathrm{C}$ (stabilized with $\pm 0.1{ }^{\circ} \mathrm{C}$ ), equipped with the ${ }^{7} \mathrm{Li} /{ }^{1} \mathrm{H}$ or ${ }^{19} \mathrm{~F}$ coil $(5 \mathrm{~mm})$. In each case, the gradient strengths were varied from $5-1800 \mathrm{G} \mathrm{cm}^{-1}$, with gradient pulse length $\delta$ of $1 \mathrm{~ms}$ and diffusion time of $50 \mathrm{~ms}$, recording 16 scans at relaxation delays of up to $60 \mathrm{~s}$. The self-diffusion coefficients were determined by fitting peak intensities as a function of the gradient according to eqn (1):

$$
I=I_{0} \cdot \mathrm{e}^{-D \cdot \gamma^{2} \cdot g^{2} \cdot \delta^{2} \cdot\left(\Delta-\frac{\delta}{3}\right)}
$$

$I, I_{0}, D$ and $g$ are the observed intensity of the NMR signal, its initial intensity, the diffusion coefficient, and the applied gradient strength. The corresponding gyromagnetic ratio $\gamma$ was set to $1.655 \times 10^{3} \mathrm{~Hz} \mathrm{G}^{-1}$ and $4.006 \times 10^{3} \mathrm{~Hz} \mathrm{G}^{-1}$ for ${ }^{7} \mathrm{Li}$ and ${ }^{19} \mathrm{~F}$. A preliminary data analysis was performed based on the Bruker Diff tool (Bruker Topspin3 software) and then refined with the Origin 2016 program package. 


\section{Raman measurements}

Raman measurements were performed with a Bruker VERTEX 70 spectrometer equipped with a RAM II Raman module and $\mathrm{Nd}$ :YAG laser with a wavelength of $1064 \mathrm{~nm}$ and power output of $300 \mathrm{~mW}$. 1000 scans were acquired at a resolution of $2 \mathrm{~cm}^{-1}$, all spectra were stored in the range from $0 \mathrm{~cm}^{-1}$ to $4000 \mathrm{~cm}^{-1}$. Bruker OPUS software was applied for the Raman measurements whereas the Origin 2016 program package was used for data analysis or peak deconvolution.

\section{Molecular dynamic simulations}

The MD simulations have been performed with the in-house developed code using the APPLE\&P polarizable force field. ${ }^{49-51}$ All systems contained 56 LiTFSI ion pairs inside a cubic simulation box with a size of $\sim 45 \AA$, corresponding to a lithium concentration of $1 \mathrm{~mol} \mathrm{~L}^{-1}$. The number of molecules of EC, DMC and $\mathrm{Pyr}_{14}$ TFSI were adjusted such that the following weight percentages of the IL were obtained: 0, 10, 30, 50 and $80 \mathrm{wt} \%$ $\mathrm{Pyr}_{14}$ TFSI. Note that unlike the available experimental data, we did not simulate electrolytes with $100 \mathrm{wt} \% \mathrm{Pyr}_{14}$ TFSI due to their quite large intrinsic viscosities (see discussion below), which would require considerable equilibration and simulation times without providing substantially new insights. The systems were equilibrated for $5 \mathrm{~ns}$ in the $N p T$ ensemble, followed by subsequent production runs of at least 30-100 ns, depending on the actual electrolyte composition, in the $N p T$ ensemble at $298 \mathrm{~K}\left(25{ }^{\circ} \mathrm{C}\right)$. Both, the temperature and the pressure of the system were maintained by a Nosé-Hoover chain thermostat ${ }^{52}$ (coupling frequency $0.01 \mathrm{fs}^{-1}$ ) and barostat (coupling frequency $0.0005 \mathrm{fs}^{-1}$ ), while periodic boundary conditions were applied in all dimensions. Electrostatic interactions have been treated by the Ewald summation technique with a cut-off radius of $15 \AA$, an inverse Gaussian charge width of $0.23 \AA^{-1}$, and $8 \times 8 \times 8$ vectors for the part in reciprocal space. Lennard-Jones interactions have been truncated at $15 \AA$, beyond which a continuum-model dispersion correction was applied. All bonds were constrained by the SHAKE algorithm. ${ }^{53,54}$ Note that a multiple-time-step integration scheme ${ }^{55,56}$ was used to integrate the equations of motion, where a time step of 0.5 fs has been used for bonds and angles, respectively. For torsions and non-bonded interactions up to a distance of $7 \AA$, a time step of 1.5 fs was used, and finally, for non-bonded interactions between atoms separated more than $7 \AA$ and the reciprocal part of the Ewald summation, a time step of 3 fs was used. The induced dipoles were determined iteratively where the corresponding dipole-dipole interactions were scaled to zero by a tapering function between 14.5 and $15 \AA$.

\section{Results and discussion}

All observable key phenomena in electrochemical cells involve the transport of charged particles (e.g., electrons or ions) between the electrodes and charge transfer at electrode surfaces. In the absence of electric fields, successful ion transport or diffusion is considered as thermally activated process originating from a series of random ion displacements as long as the relevant transport process is not related to cooperative motions of molecular segments (such as e.g. polymer chains). ${ }^{21,22,57-60}$ In contrast to proton conduction in aqueous phases, which is often based on vehicular diffusion or Grotthuss-like "hopping", of hydrated hydronium ions, ${ }^{23,26,61}$ the presence of various solvents and additives in addition to lithium salts and/or ILs renders an explicit description of probable mechanisms and phenomena responsible for an observable charge transport in lithium ion batteries difficult. Nevertheless, the nature of available lithium species and its surrounding "liquid" structure is often accessible from MD simulations thus facilitating identification of complex lithium ion transfer mechanisms in liquid electrolytes. ${ }^{27,62}$ In diluted liquid solutions, the lithium salt may fully dissociate into solvated lithium ions and anions while in case of more concentrated solutions, anions could possibly attach to the lithium ions yielding aggregates including ion pairs, triple ions or even so-called quadrupoles, respectively. ${ }^{63}$ Such lithium coordination species may be distinguished based on e.g. their molecular interactions and rotational dynamics that occur in the picosecond-nanosecond time range. ${ }^{64}$ In addition, Raman spectroscopy constitutes an indispensable tool to actually determine the ion coordination, ${ }^{65}$ though the presence of multiple species in ternary electrolyte solutions that are Raman active makes it quite challenging to analyze the underlying molecular coordination due to possible overlap of the individual peaks. For this reason, we complemented obtained Raman data by MD simulation to describe the lithium coordination in more detail, including a discussion of transport coefficients (from diffusion and conductivity data). Subsequently, we explained the experimental data using a microscopic analysis of correlated motions of individual ion species, particularly focusing on the role of the electrolyte's viscosity.

\section{Ion coordination}

In the Raman spectra of the corresponding electrolytes, we observed a peak at $743 \mathrm{~cm}^{-1}$ attributed to $\mathrm{PF}_{6}{ }^{-}$, which is covered by TFSI $^{-}$peaks and therefore is only clearly discernible if no $\mathrm{Pyr}_{14}$ TFSI is present in the electrolyte. Assuming that the peak of $\mathrm{PF}_{6}{ }^{-}$remains unchanged (see discussion below), it was subtracted from other Raman spectra. An additional peak overlap affects the C-O stretching vibration of EC and DMC at 900-940 $\mathrm{cm}^{-1}$ where $\mathrm{Pyr}_{14}{ }^{+}$ions also exhibit peaks, so that the peaks reflecting $\mathrm{Li}^{+}-\mathrm{EC}$ and $\mathrm{Li}^{+}$-DMC coordination can hence only be unambiguously identified if $\mathrm{Pyr}_{14}{ }^{+}$interacts weakly with EC and DMC. This assumption is verified by $\mathrm{MD}$ simulation results, from which we observed that the radial distribution functions (RDFs) between the centers of mass of $\mathrm{Pyr}_{14}{ }^{+}$and EC as well as of $\mathrm{Pyr}_{14}{ }^{+}$and DMC show only a weak first coordination peak with a magnitude of about 1.3-1.5. After deconvolution of the Raman spectra and subtracting peaks attributed to either $\mathrm{Pyr}_{14}{ }^{+}$or $\mathrm{PF}_{6}{ }^{-}$ions, the average coordination numbers $n$ can be determined by multiplying the ratio of the ligand (e.g., $\mathrm{TFSI}^{-}, \mathrm{EC}$ or DMC) concentration $c_{\text {Ligand }}$ and the $\mathrm{Li}^{+}$ concentration $c_{\mathrm{Li}+}$ with the ratio of the integrated peak areas:

$$
n=\frac{c_{\text {Ligand }}}{c_{\mathrm{Li}^{+}}} \frac{A_{\text {coord }}}{A_{\text {coord }}+A_{\text {free }}}
$$


Here, $A_{\text {coord }}$ is the peak area attributed to the coordinating ligand, while $A_{\text {free }}$ denotes the peak area of "free" TFSI ${ }^{-}$, EC or DMC. In order to apply eqn (2), we considered that "free" and coordinated ligands more or less have comparable Raman scattering coefficients and that each ligand coordinates to a single $\mathrm{Li}^{+}$cation only. ${ }^{66}$ The latter can readily be verified on the basis of the MD simulations, where we identified in case of all IL concentrations that the fraction of ligand molecules coordinating to two lithium ions simultaneously - a situation commonly encountered for TFSI $^{-}$involving four coordinating oxygen sites - is on the order of a few percent. The coordination numbers derived from the Raman data are given in Fig. 1 including coordination numbers extracted from MD simulations. They reveal that the lithium ions are moderately coordinated to $\mathrm{PF}_{6}{ }^{-}$anions (on average ca. $0.4 \mathrm{PF}_{6}{ }^{-}$per $\mathrm{Li}^{+}$), roughly independent of the $\mathrm{Pyr}_{14}$ TFSI concentrations (see lower panel of Fig. 1), thus justifying the subtraction of the $\mathrm{PF}_{6}{ }^{-}$peak from the remaining spectra. Moreover, due to the rather weak influence of $\mathrm{PF}_{6}{ }^{-}$, it appears reasonable that the coordination numbers of the remaining ligands do not change significantly upon substitution of the conducting salt. However, the situation changes in the limit of higher IL fractions where a crossover from carbonate-based lithium coordination to purely anionbased coordination is observed (Fig. 1). Indeed, Raman spectra of electrolytes containing $100 \mathrm{wt} \% \mathrm{Pyr}_{14}$ TFSI and LiTFSI suggest strong coordination between $\mathrm{Li}^{+}$and $\mathrm{TFSI}^{-}$, which is partially suppressed in cases where a second anion species $\left(\right.$ e.g., $\left.\mathrm{PF}_{6}{ }^{-}\right)$is present (as observed from the spectrum of $\mathrm{Pyr}_{14} \mathrm{TFSI}^{\mathrm{T}} \mathrm{LiPF}_{6}-$ electrolyte). Thus, employing $\mathrm{LiPF}_{6}$ as conducting salt partly weakens the present Li-TFSI-interactions yielding larger fractions of "free" TFSI" anions and a more compact lithium solvation shell due to the smaller molecular size of $\mathrm{PF}_{6}{ }^{-}$anions, which in principle could enhance the lithium ion transport (see below).

The C-O stretching vibration bands of EC $\left(\sim 894 \mathrm{~cm}^{-1}\right)$ and DMC $\left(\sim 917 \mathrm{~cm}^{-1}\right)$ shifted to higher wavenumbers $\left(\sim 905 \mathrm{~cm}^{-1}\right.$ and $935 \mathrm{~cm}^{-1}$ ) upon interaction of $\mathrm{Li}^{+}$with solvent molecules. An analysis of these four Raman bands showed that lithium is coordinated by 2.2 EC molecules in agreement with the MD simulation data. Conversely, the Raman spectra indicate that on average 0.9 DMC molecules coordinate a lithium ion in the IL-free electrolyte, while MD data reveal a substantially higher coordination number of 1.6 DMC molecules. This difference is attributed to the fact that DMC can attain different conformers and coordinate lithium ions in different ways. Based on DFT calculations it was demonstrated that DMC could bind to $\mathrm{Li}^{+}$ with its carbonyl oxygen in either cis-cis (DMCcc) or cis-trans (DMCct) conformation (with respect to its methoxy groups), where each coordination state is reflected by different Raman frequencies shifted by $\sim 60-70 \mathrm{~cm}^{-1}$, mainly as resulting from different dipole moments. ${ }^{67}$ Apart from that, DMC may also coordinate $\mathrm{Li}^{+}$by its two ethylene oxide oxygen atoms in cis-cis conformation. Here, DFT calculations revealed that the two motifs exhibit different Raman bands, of which the Raman peak of the ethylene-oxygen coordination is located close to the frequency of uncoordinated EC. ${ }^{67}$ Notably, from analysis of MD data regarded as representative of the considered electrolytes,
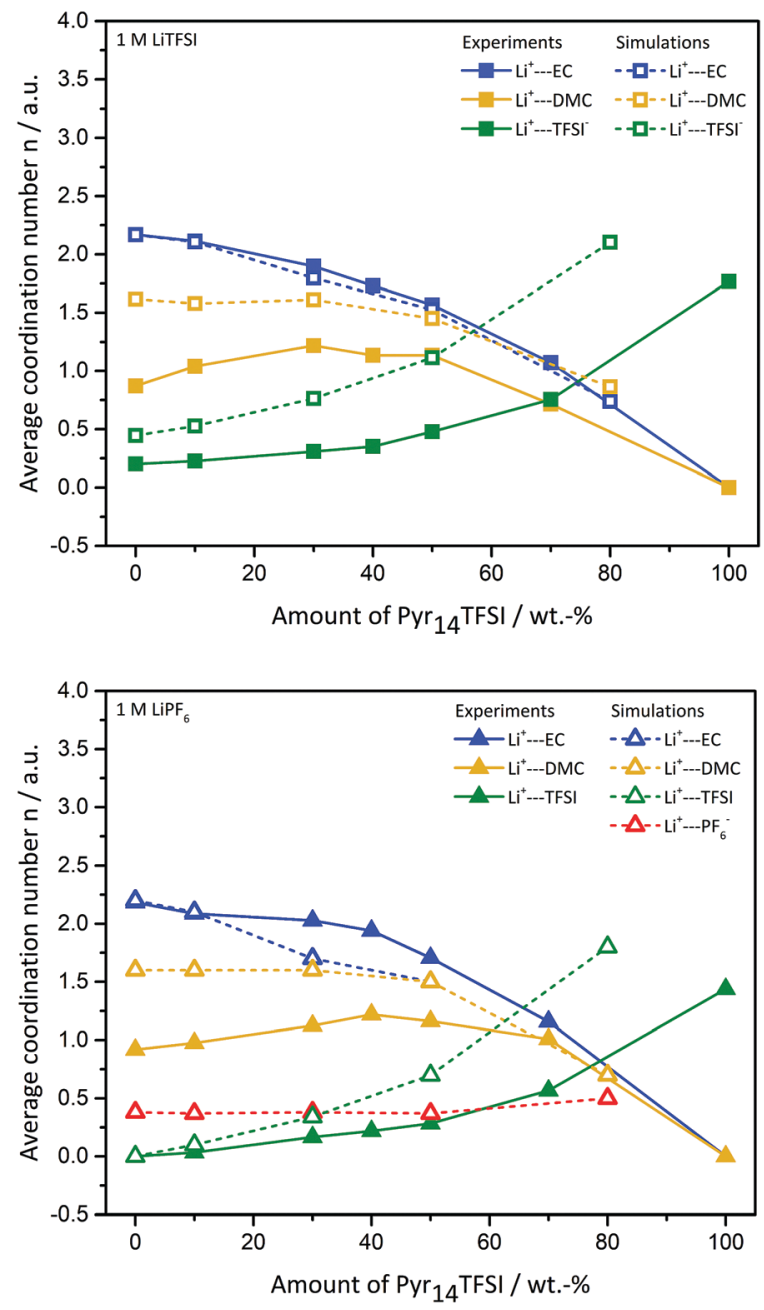

Fig. 1 Coordination numbers of lithium coordinated by different species present in blends of $\mathrm{Pyr}_{14}$ TFSI, EC and DMC with $1 \mathrm{M}$ LiTFSI (top) and $1 \mathrm{M}$ $\mathrm{LiPF}_{6}$ (bottom) as conducting salts at $25^{\circ} \mathrm{C}$. $\mathbf{\Delta}$ Experimental data points. $\square \triangle$ Simulation data points. The solid and dashed lines are guides to the eye. The size of the data points represents the error of the measurements and calculations.

it was found that ethylene-oxygen coordination is of minor importance (i.e., reflecting only a few percent of the overall $\mathrm{Li}^{+}$-DMC coordination), though the situation can be slightly different at higher lithium concentrations. ${ }^{68}$ Nonetheless, in addition to frequency shifts upon $\mathrm{Li}^{+}$coordination, the computed Raman activity of coordinating EC moieties is roughly $20 \%$ larger than that of DMC molecules coordinated in cis-cis conformation, ${ }^{67}$ thus rationalizing deviations between the Raman data and simulation results in Fig. 1. Despite these deviations, our Raman results are similar to previously reported data ${ }^{39,69}$ rendering the error margins of the obtained coordination numbers (see Fig. 1) rather negligible. Also, a detailed inspection of the most probable solvation shell(s) of $\mathrm{Li}^{+}$in our simulations for purely carbonate-based clusters revealed favored interactions among $\mathrm{EC}$ and $\mathrm{Li}^{+}$over DMC, in excellent agreement with reported $a b$ initio MD simulations. ${ }^{67}$ Note that even if DMC was replaced by 1,2-dimethoxy-ethane (DME), which due to its 
bidentate character is supposed to coordinate much stronger to $\mathrm{Li}^{+}$, EC was still found to preferentially coordinate lithium ions. ${ }^{69}$

Indeed, both EC and DMC coordination numbers decrease in the presence of higher fractions of $\mathrm{Pyr}_{14}$ TFSI, as the carbonates in the lithium solvation shell are successively replaced by TFSI $^{-}$ anions. Here, the TFSI $^{-}$anions strongly coordinate to lithium ions but rather weakly to sterically shielded $\mathrm{Pyr}_{14}{ }^{+}$cations. ${ }^{70}$ Note that the Raman peak at $\sim 740 \mathrm{~cm}^{-1}$ constitutes the most suitable peak for analyzing $\mathrm{Li}^{+}-\mathrm{TFSI}^{-}$interactions. ${ }^{39,66,70-72}$ No shift is observed for "free" TFSI $^{-}$(fully solvated by weakly interacting $\mathrm{Pyr}_{14}{ }^{+}$cations) while coordinated $\mathrm{TFSI}^{-}$(ion pairs or aggregates) shifted to higher wavenumbers $\left(\sim 748 \mathrm{~cm}^{-1}\right)$. The coordination numbers obtained from Raman peak deconvolution are also given in Fig. 1, indicating that higher amounts of Pyr $_{14}$ TFSI lead to an increasing average coordination number of $\mathrm{TFSI}^{-}$. In the case of an excess of organic carbonates in the electrolytes, the sum of the average coordination numbers of TFSI ${ }^{-}$, EC and DMC for each mixture is roughly four, reflecting that tetrahedral coordination may be preferred for non-transition metal complexes. ${ }^{73}$ Coordination numbers of 1.5-2 for TFSI $^{-}$anions in blends with $100 \mathrm{wt} \%$ IL suggest that two oxygen atoms from each TFSI $^{-}$ion coordinate to lithium ions (bidentate coordination), thereby allowing the lithium ion to adopt the favored fourfold coordination. In addition, lithium ions preferentially bind to EC and DMC solvent molecules, which can be seen from the shape of the curves in Fig. 1 as compared to a linear dependence with the IL mole fraction expected for ideal mixtures. The $\mathrm{Li}^{+}-\mathrm{TFSI}^{-}$interaction is suppressed by coordination of lithium ions to organic carbonate molecules, which is in agreement with reported data for EMImTFSI where the addition of EC to EMImTFSI yielded higher fractions of "free" TFSI $^{-}$anions. ${ }^{74}$

In the following, we considered the coordination of lithium ions and TFSI anions in more detail. For monodentate coordination, one would expect a Raman peak at $746 / 747 \mathrm{~cm}^{-1}$, whereas bidentate or other coordination would be revealed by peaks at $748 / 749 \mathrm{~cm}^{-1}$ and 749-750/750-752 $\mathrm{cm}^{-1}$, respectively. ${ }^{75}$ However, merely a single peak was recognized indeed reflecting the sum of all possible TFSI coordination motifs, so that the coordination numbers derived from the experiments refer to both monodentate and bidentate coordination. In this case, the MD simulations provided further insights, since they allowed direct study of different coordination modes, e.g. by analyzing the RDFs between lithium ions and nitrogen atoms of the TFSI $^{-}$anions (ESI $\dagger$ ). Apart from a minor first peak attributed to the direct coordination of nitrogen atoms, a double-peak structure is visible for the first coordination shell (up to $\sim 5 \AA$ ), arising from coordination of the TFSI $^{-}$oxygen atoms to lithium ions. Explicitly, the first peak reflects bidentate binding of TFSI $^{-}$(resulting in slightly shorter equilibrium distances), whereas the second peak may be attributed to monodentate TFSI $^{-}$coordination (yielding slightly larger $\mathrm{Li}-\mathrm{N}$ distances). The populations of the distinct coordination motifs can be estimated by taking the volume integral of the two peaks, thereby revealing that the fraction of bidentate $\mathrm{TFSI}^{-}$coordination increases with higher amounts of $\mathrm{Pyr}_{14}$ TFSI, starting from roughly equal fractions of the two coordination types for $0 \mathrm{wt} \% \mathrm{Pyr}_{14}$ TFSI up to almost $60 \%$ bidentate coordination for $80 \mathrm{wt} \% \mathrm{IL}$. Thus, the bidentate TFSI $^{-}$coordination remains dominant in all considered electrolytes. Notably, similar trends can be derived from the experimental Raman spectra, where the second peak shifts to higher wavenumbers. The relative free energy difference required to change lithium ions from mono- to bidentate coordination based on the respective probabilities mentioned above was estimated to $\sim-0.65 \mathrm{~kJ} \mathrm{~mol}^{-1}$ in the limit of high IL concentrations, a value well below the thermal energy. This finding suggests rapid interchange between the two coordination motifs. Despite that the coordination motifs of the considered lithium ions, that were established based on Raman and MD data, reflect a delicate balance of molecular interactions with solvent molecules or of other ions with lithium ions, they indeed allow for advanced understanding of the achievable macroscopic charge transport in the respective IL/carbonate solvent electrolyte blends, and thus may pave the way for further improvement of future electrolytes.

\section{Self-diffusion coefficients}

The experimentally obtained self-diffusion coefficients $D$ for all ionic species comprising the considered electrolytes are summarized in Table 1, reflecting typically observed values for viscous materials. The slightly larger diffusion coefficients for lithium ions in case of

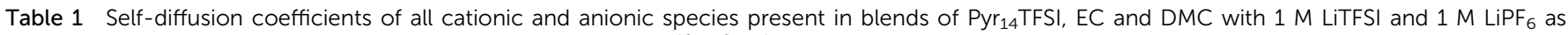
conducting salts at $25^{\circ} \mathrm{C}$. All data is accurate within $\pm 0.2 \times 10^{-10} \mathrm{~m}^{2} \mathrm{~s}^{-1}$

\begin{tabular}{|c|c|c|c|c|c|c|c|c|c|}
\hline & Amount of $\mathrm{Pyr}_{14} \mathrm{TFSI} / \mathrm{wt} \%$ & \multicolumn{2}{|c|}{$\underline{\mathrm{D}\left(\mathrm{Li}^{+}\right) / 10^{-10} \mathrm{~m}^{2} \mathrm{~s}^{-1}}$} & \multicolumn{2}{|c|}{$\underline{\mathrm{D}\left(\operatorname{Pyr}_{14}{ }^{+}\right) / 10^{-10} \mathrm{~m}^{2} \mathrm{~s}^{-1}}$} & \multicolumn{2}{|c|}{$\underline{\mathrm{D}\left(\mathrm{TFSI}^{-}\right) / 10^{-10} \mathrm{~m}^{2} \mathrm{~s}^{-1}}$} & \multicolumn{2}{|c|}{$\underline{\mathrm{D}\left(\mathrm{PF}_{6}^{-}\right) / 10^{-10} \mathrm{~m}^{2} \mathrm{~s}^{-1}}$} \\
\hline \multirow[t]{4}{*}{ Experiments } & 0 & 2.0 & 1.7 & - & - & - & 2.1 & 3.0 & - \\
\hline & 30 & 1.1 & 0.9 & 0.9 & 1.1 & 1.3 & 1.1 & 1.5 & - \\
\hline & 40 & 0.8 & 0.7 & 0.8 & 0.8 & 0.9 & 0.8 & 1.0 & - \\
\hline & 50 & 0.5 & 0.5 & 0.5 & 0.6 & 0.6 & 0.6 & 0.6 & - \\
\hline \multirow[t]{5}{*}{ Simulations } & 0 & 2.6 & 2.3 & - & - & - & 2.6 & 3.3 & - \\
\hline & 10 & 1.6 & 1.8 & 2.2 & 1.8 & 2.2 & 2.1 & 2.1 & - \\
\hline & 30 & 1.1 & 1.2 & 1.1 & 1.1 & 1.3 & 1.2 & 1.5 & - \\
\hline & 50 & 0.6 & 0.6 & 0.6 & 0.6 & 0.6 & 0.6 & 0.7 & - \\
\hline & 80 & 0.1 & 0.1 & 0.1 & 0.2 & 0.1 & 0.1 & 0.1 & - \\
\hline
\end{tabular}


$\mathrm{LiPF}_{6}$ could partly be related to the somewhat smaller size of the overall lithium-ion solvation shell when $\mathrm{PF}_{6}{ }^{-}$rather than TFSI is involved in the coordination shell. The corresponding apparent self-diffusion coefficients were also derived from MD simulation trajectories via the Einstein relation:

$$
D_{i}=\lim _{\Delta t \rightarrow \infty} \frac{\left\langle\Delta r_{i}^{2}(\Delta t)\right\rangle}{6 \Delta t},
$$

where the subscript $i$ denotes the index of the molecule species and $\left\langle\Delta r_{i}^{2}(\Delta t)\right\rangle$ the mean squared displacement of the molecules during the time interval $\Delta t$. An analytical correction for the finite size of the simulation box of the form:

$$
\Delta D_{\mathrm{FSC}}=\frac{2.837 k_{\mathrm{B}} T}{6 \pi \eta L}
$$

was applied, ${ }^{76-78}$ due to the fact that the long-range hydrodynamic interactions contributing to overall diffusivity are not quantitatively reproduced in finite size simulation systems. Here, $L$ is the length of the simulation box, while (partly interpolated) experimental values were utilized for the viscosity $\eta$. Note that the magnitude of the correction was determined for a majority compound (that is either the average of EC and DMC or TFSI). Subsequently, all diffusion coefficients were scaled by the same ratio between corrected and observed diffusion coefficients for this compound, where the correction is in the range of $5-10 \%$. As anticipated, the self-diffusion coefficients of all species decrease with higher amounts of $\mathrm{Pyr}_{14}$ TFSI. The experimental and simulated data are in reasonable agreement for the charged species, but exhibit slight differences for uncharged species.

\section{Ionic conductivities}

Since self-diffusion coefficients include contributions of noncharged species such as bulk motion of ion pairs or higher ion aggregates with no resulting net charge, we determined the ionic conductivities and transport numbers of the considered materials using both impedance and PFG NMR measurements. The comparison of ionic conductivities from impedance measurements with those based on PFG NMR self-diffusion coefficients in principle yields valuable information on the relative importance of ionic correlations, as expressed by the ratio of impedance and PFG NMR based conductivities that reveals the degree of ion dissociation (or association) within the considered electrolytes. The results from impedance measurements are shown in Fig. 2 for blends with 0, 30 and $70 \mathrm{wt} \% \mathrm{Pyr}_{14}$ TFSI. All other data are shown in ESI. $\dagger$ We note that both the $\mathrm{LiPF}_{6}{ }^{-}$and LiTFSI-based IL/carbonate blends show highly comparable conductivities for all ratios in the entire temperature range, though blends with LiPF $_{6}$ show slightly higher conductivities. All ionic conductivities increase with decreasing amounts of Pyr $_{14}$ TFSI as well as with increasing temperature following a Vogel-Tammann-Fulcher (VTF) type behavior (i.e., $\sigma=\sigma_{0} \exp \left(-B /\left(T-T_{0}\right)\right.$ ), with $B$ being related to a pseudo-activation energy by $B \cdot R ; R$ is the molar gas constant, and $T_{0}$ denotes a characteristic temperature of the system). Compared to a standard aqueous $0.1 \mathrm{M} \mathrm{KCl}$ solution, the ionic conductivities of IL/carbonate

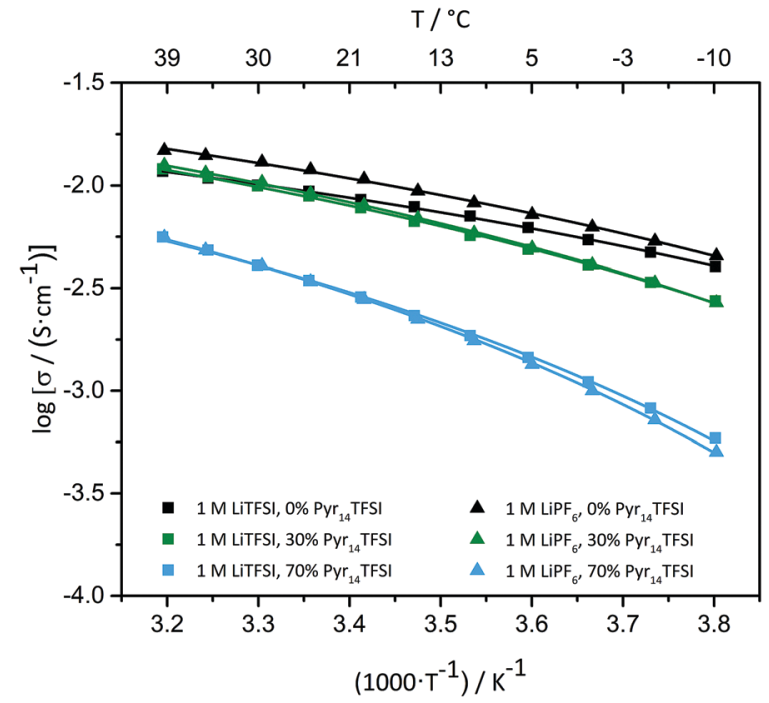

Fig. 2 Arrhenius plot of ionic conductivities of $\mathrm{Pyr}_{14}$ TFSI/EC/DMC blends $\left(0,30\right.$ and $\left.70 \mathrm{wt} \% \mathrm{Pyr}_{14}{ }_{14 F S I}\right)$ with $1 \mathrm{M}$ LiTFSI or $1 \mathrm{M} \mathrm{LiPF}_{6}$ as conducting salts measured from -10 to $40{ }^{\circ} \mathrm{C}$ using impedance. The data points are fitted by VTF functions (solid lines).

blends are rather low, ${ }^{79}$ since a conductivity of $1.17 \times 10^{-2} \mathrm{~S} \mathrm{~m}^{-1}$ for blends without ionic liquid at $40{ }^{\circ} \mathrm{C}$ is already reached by the $\mathrm{KCl}$ solution around $20{ }^{\circ} \mathrm{C}$. The obtained ionic conductivity of the standard solution at $0{ }^{\circ} \mathrm{C}$ is $7.1 \times 10^{-3} \mathrm{~S} \mathrm{~cm}^{-1}$ and therefore more than five times higher than the corresponding ionic conductivity of $100 \mathrm{wt} \%$ IL at $40{ }^{\circ} \mathrm{C}\left(1.3 \times 10^{-3} \mathrm{~S} \mathrm{~cm}^{-1}\right)$. In order to fit the curves in Fig. 2, $T_{0}$ values were approximated by $T_{\mathrm{g}}$ values estimated from differential scanning calorimetry (DSC) measurements (see ESI, $\dagger$ with $T_{\mathrm{g}}$ being the glass transition temperature of the system), though conceptually $T_{0}$ is neither identical nor related to $T_{\mathrm{g}}$ in a very simple manner. ${ }^{80}$ We nonetheless applied this approximation due to the fact that the curves for the electrolytes with a low IL content in Fig. 2 are only marginally curved, for which a VTF fit with $T_{0}$ as a separate fitting parameter would be somewhat ambiguous. When comparing the IL-free electrolyte with the electrolyte with $100 \mathrm{wt} \% \mathrm{IL}, T_{0}$ increases from $\sim 175 \mathrm{~K}$ to $\sim 198 \mathrm{~K}$, independent of the employed conducting salt (i.e. $\mathrm{LiPF}_{6}{ }^{-}$or LiTFSI). Additionally, pseudo-activation energies $B \cdot R$, which may be extracted from both viscosity and conductivity data, were determined. In both cases, $T_{0}$ values estimated from DSC data have been used for VTF fits. The obtained pseudoactivation energies are summarized in the ESI. $\dagger$ Note that the values display a marginal increase within the uncertainties at low and intermediate $\mathrm{Pyr}_{14}$ TFSI concentrations (roughly up to $50 \mathrm{wt} \% \mathrm{IL})$, followed by a steeper increase in the limit of high $\mathrm{Pyr}_{14}$ TFSI fractions. This means that the effective barrier for the ion transport increases, and that larger IL concentrations result in an impaired ion transport. For blends with compositions in the range of $0-40 \mathrm{wt} \% \mathrm{Pyr}_{14}$ TFSI, the pseudo-activation energies derived from impedance data are in good agreement with the respective energies calculated from the viscosity data. In the range of $50-100 \mathrm{wt} \% \mathrm{IL}, B_{\mathrm{Visco}} \cdot R$ exceeds $B_{\mathrm{Imp}} \cdot R$ by up to $30 \%$, suggesting that the overall molecular motion is more hindered compared to the motion of charge carriers only. In general, the 
pseudo-activation energies are comparable for both conducting salts, though the values in the limit of high $\mathrm{Pyr}_{14}$ TFSI concentrations appear to be slightly larger in blends containing LiPF $_{6}$. Since no significant difference between both conducting salts could be observed in this regime (see Table 1 or Fig. 2), this unambiguously reflects uncertainties in the fitting procedure outlined above.

In addition to experimental ionic conductivities obtained from impedance measurements, we have also estimated the ideal molar conductivities (i.e., if no ion correlations would be present, reflecting a Haven ratio of 1) from the PFG NMR data via the Nernst-Einstein equation:

$$
\sigma_{\mathrm{NMR}}=\frac{N_{\mathrm{A}} \cdot e^{2} \cdot \sum\left(\left(z_{i}\right)^{2} c_{i} D_{i}\right)}{k_{\mathrm{B}} \cdot T}
$$

Here, $N_{\mathrm{A}}, e$ and $k_{\mathrm{B}}$ are the Avogadro constant, the elementary charge, and the Boltzmann constant, whereas $z_{i}, c_{i}$ and $D_{i}$ are the charge, the concentration and the diffusion coefficient of the ionic species $i$, respectively. Note that deviations from the ideal behavior expressed by eqn (5) can in principle be quantified by various formalisms, such as eqn (6) and the degree of ion dissociation (see below) or alternatively the Haven ratio. ${ }^{81}$ Hence, $\sigma_{\mathrm{NMR}}$ serves as a reference for the actual conductivities. Furthermore, molar ionic conductivities were derived from MD simulation data, where similarly to the experimental characterization, the ionic conductivity reflecting macroscopic mass transport (including motion of neutral ion pairs or aggregates but otherwise neglecting contributions from complex correlated ion motion) as monitored by PFG NMR and the primarily charge transport (in agreement with the ionic conductivity determined from impedance data) have been computed (Fig. 3). The latter quantity was extracted from simulations via a generalized expression of eqn (5):

$$
\sigma=\lim _{\Delta t \rightarrow \infty} \frac{e^{2}}{6 k_{\mathrm{B}} T V \Delta t} \sum_{i=1}^{N} \sum_{j=1}^{N} z_{i} z_{j}\left\langle\Delta r_{i}(\Delta t) \cdot \Delta r_{j}(\Delta t)\right\rangle,
$$

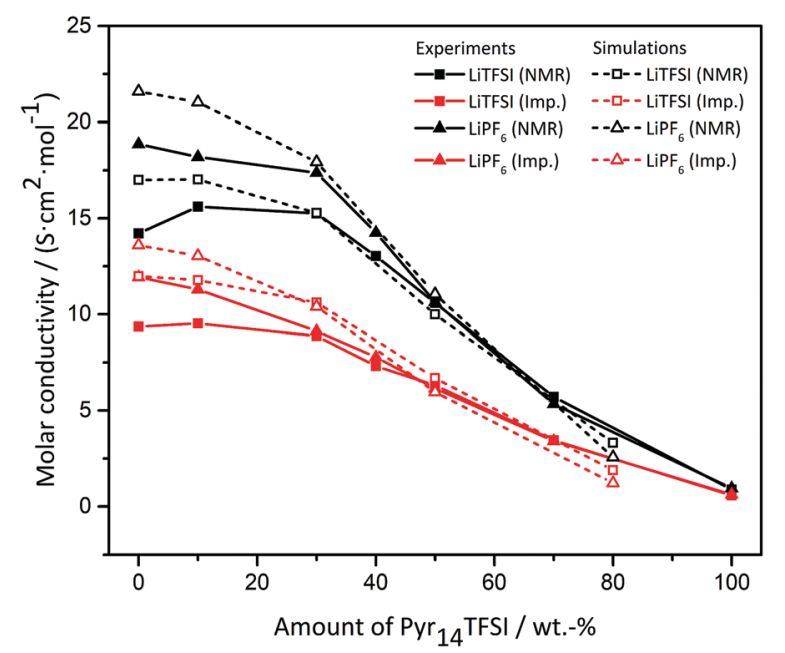

Fig. 3 Molar conductivities of $\mathrm{Pyr}_{14}$ TFSI/EC/DMC blends with 1 M LiTFSI or $1 \mathrm{MLiPF}_{6}$ as conducting salts at $25{ }^{\circ} \mathrm{C}$ derived from PFG NMR and impedance measurements. $\mathbf{\Delta}$ Experimental data points. $\square \triangle$ Simulation data points. The solid and dashed lines are guides to the eye. where $V$ denotes the volume of the simulation box containing $N$ ions in total, and $\Delta t$ is the time interval for which the displacement vectors $\Delta r_{i}$ of the individual ions are calculated. Note that in contrast to eqn (5), the two summations run over the individual ions in the simulation box rather than the number of ion species present in the system. In practice, $\Delta t$-values in the range of $300 \mathrm{ps}$ to $10 \mathrm{~ns}$ have been used to assure a reasonable convergence of the averages in eqn (6) to their long-time limits, which at the same time still contain sufficient statistics. Eqn (6) also illustrates the way in which dynamical ion correlations affect the transport: if both the cation and anion move cooperatively into the same direction (reflected by $\left(\left\langle\Delta r_{i}(\Delta t) \cdot \Delta r_{j}(\Delta t)\right\rangle>0\right)$ ), their contribution to the ionic conductivity $\sigma$ becomes negative due to the fact that $z_{i} z_{j}<0$ in this case. For the subsequent discussion, however, it is important to emphasize that no structural information (such as e.g. ion coordination discussed above) enters eqn (6).

Using $1 \mathrm{M}$ LiTFSI in a given solvent blend yields a maximum molar ionic conductivity of $9.5 \mathrm{~S} \mathrm{~cm}^{2} \mathrm{~mol}^{-1}$ (impedance) and $15.6 \mathrm{~S} \mathrm{~cm}^{2} \mathrm{~mol}^{-1}$ (NMR) for an IL fraction of $10 \mathrm{wt} \% \mathrm{Pyr}_{14}$ TFSI. This may be ascribed to the presence of the additional ionic species $\mathrm{Pyr}_{14}{ }^{+}$and thus the increased amount of total charge carriers. However, higher amounts of IL result in lower ionic conductivities due to the viscosity increase, leading to a slowdown of the dynamics of the entire systems. In the presence of $\mathrm{LiPF}_{6}$, the actual molar conductivities are constantly decreasing with increasing amounts of $\mathrm{Pyr}_{14}$ TFSI. The highest molar conductivities are therefore reached without IL $\left(18.9 \mathrm{~S} \mathrm{~cm}^{2} \mathrm{~mol}^{-1}\right.$ and $11.9 \mathrm{~S} \mathrm{~cm}^{2} \mathrm{~mol}^{-1}$ ), which may be attributed to the fact that $\mathrm{PF}_{6}{ }^{-}$is a fast diffusing species, thus the conductivity of the pure organic carbonate electrolyte exceeds the maximum conductivity of all IL blend compositions (see also the respective anionic diffusion coefficients for $0 \mathrm{wt} \% \mathrm{Pyr}_{14}$ TFSI, Table 1). Note that the substitution of the conducting salt will only have an impact on molar conductivities if an excess of carbonates is present. Higher IL concentrations reduce this effect, so that $100 \mathrm{wt} \%$ IL leads to comparable values of the molar conductivities for both salts $\left(0.9 \mathrm{~S} \mathrm{~cm}^{2} \mathrm{~mol}^{-1}\right.$ and $\left.0.6 \mathrm{~S} \mathrm{~cm}^{2} \mathrm{~mol}^{-1}\right)$, though their relative ratio remains nearly constant. As depicted in Fig. 3, a good agreement between experimental results and MD data for both NMR and impedance based ionic conductivities could be observed, further validating our numerical approach, thereby allowing us to make more precise statements on specific ion correlations on the basis of eqn (6). To unravel the most probable microscopic charge transport mechanism(s) within the IL/carbonate blends, we considered various aspects of underlying ion correlation, followed by a discussion of viscosity-related effects.

\section{Degree of ion dissociation}

As mentioned above, impedance measurements are susceptible to the overall charge transport, which is typically strongly affected by dynamical ion correlations that tend to decrease the net motion of charges. In contrast, PFG NMR measures the average mass transport of the individual molecular species, which usually exceeds the bare charge transport, since except for motion of ion pairs and higher aggregates, cooperative ion motion is not captured by this experimental technique. To quantify this disparity, we 
compute the ratio between "real" conductivity, $\sigma_{\text {Impedance, }}$ and ideal conductivity $\sigma_{\mathrm{NMR}}$, which is usually termed as the degree of ion dissociation $\alpha$ (note that this quantity basically contains the same information as the Haven ratio ${ }^{81}$ )

$$
\alpha=\frac{\sigma_{\text {Impedance }}}{\sigma_{\mathrm{NMR}}}
$$

The $\alpha$-values as a function of the IL concentration are shown in the ESI. $\dagger$ Both the experimental results and simulation data indicate that $\alpha$ is approximately independent of the amount of Pyr $_{14}$ TFSI or the employed conducting salt (i.e., LiTFSI or $\mathrm{LiPF}_{6}$ ), and $\alpha$ varies around 0.6 , indicating a moderate degree of ion association. Note that the Haven ratio $(1 / \alpha)$ then amounts to 1.67, a value typically observed for both ionic liquids and organic carbonate-based electrolytes. A Haven ratio larger than unity indicates that ions of dissimilar charge preferentially move into the same direction, i.e. when transport of ion pairs is relevant, which may be the case even at lower conducting salt concentrations. $^{37,82}$

\section{Transference and transport numbers}

Transport numbers (as opposed to the transference numbers to be discussed below) measure the contribution of a molecular species to the overall mass transport in the electrolyte, irrespective of the (effective) charge it carries. This dimensionless parameter is expressed as the fraction of the concentrationweighted diffusion coefficient $D_{i}$ of a given species relative to the sum of the diffusion coefficients of all considered species. ${ }^{83}$ For instance, the transport number $t_{i}^{\mathrm{NMR}}$ of species $i$ is given by:

$$
t_{i}^{\mathrm{NMR}}=\frac{c_{i} D_{i}}{\sum\left(\left(z_{j}\right)^{2} c_{j} D_{j}\right)}
$$

In contrast, the transference number $t_{i}^{\text {current }}$ is defined as the fraction of current $I_{i}$ carried by a given ionic species relative to the total current $I_{0}$ :

$$
t_{i}^{\text {current }}=\frac{I_{i}}{I_{0}}
$$

In the dilute limit, transport numbers and transference numbers are identical due to the absence of ion correlations. In practice, however, transport numbers of lithium ions usually exceed transference numbers, mainly due to significant interactions between cations and anions, which show correlated motion into the same direction. ${ }^{84}$ Unfortunately, the potentiostatic polarization method introduced by Bruce and Vincent, ${ }^{85}$ usually employed to determine transference numbers experimentally, cannot be applied for the present blends, as the method is established for electrolyte solutions with one dissociated salt and therefore two charged species. Because of the use of ionic liquids as (co-)solvents, our electrolytes contain more than two charged species, all of which in principle may show correlated motion. Thus, transference numbers were exclusively calculated from simulation data, whereas for experimental samples only the transport numbers were extracted on the basis of the PFG NMR data. The transport numbers of all charged species are depicted in Fig. 4. For blends containing LiTFSI as conducting salt, we note
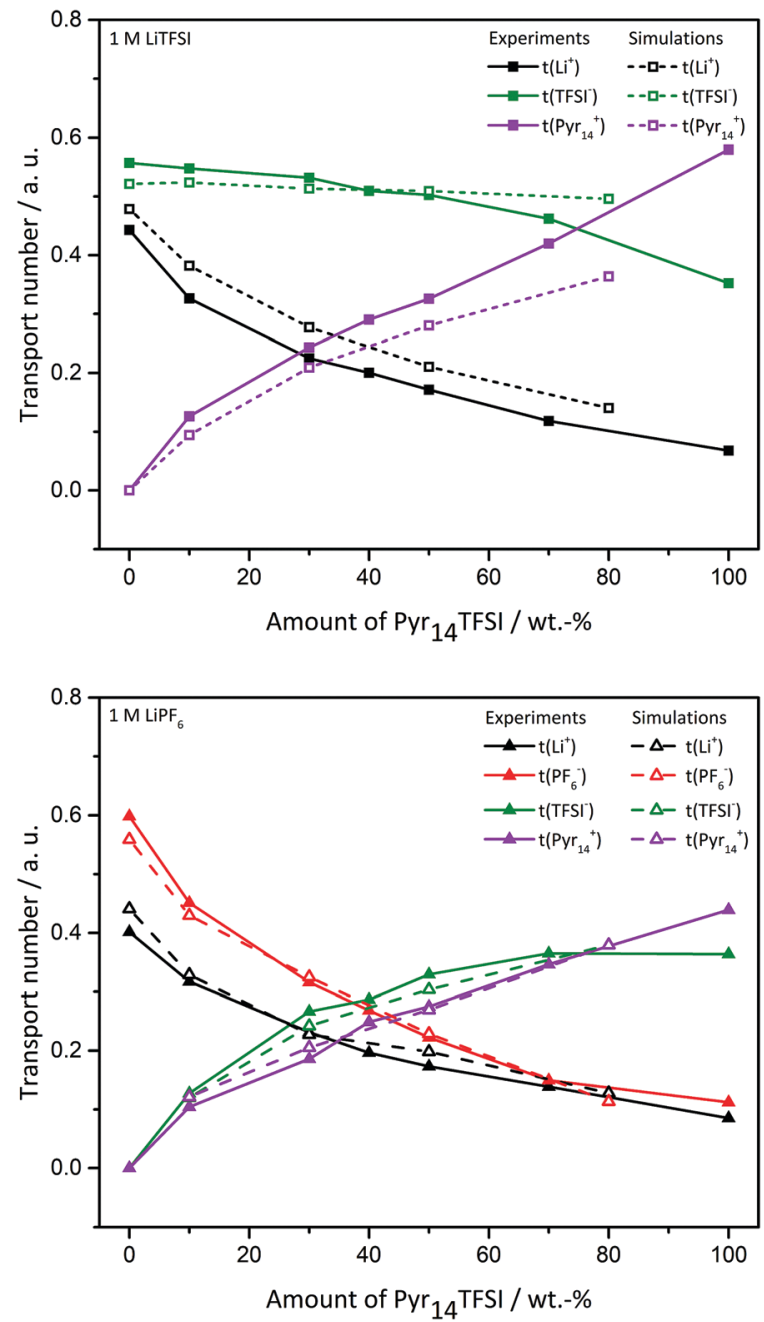

Fig. 4 Transport numbers of all ionic species in $\mathrm{Pyr}_{14}{ }_{14} \mathrm{TSSI} / \mathrm{EC} / \mathrm{DMC}$ blends with $1 \mathrm{M} \mathrm{LiTFSI} \mathrm{(top)} \mathrm{and} 1 \mathrm{M} \mathrm{LiPF}_{6}$ (bottom) as conducting salts at $25^{\circ} \mathrm{C}$. - $\Delta$ Experimental data points. $\square \triangle$ Simulation data points. The solid and dashed lines are guides to the eye.

that $t^{\mathrm{NMR}}\left(\mathrm{Pyr}_{14}{ }^{+}\right)$continuously increases to a value of about 0.6 with increasing amount of IL. Simultaneously, $t^{\mathrm{NMR}}\left(\mathrm{Li}^{+}\right)$ decreases from $\sim 0.4$ to 0.1 , showing that the lithium ions marginally contribute to the overall charge transport in the limit of high Pyr $_{14}$ TFSI concentrations as observed previously for binary IL/lithium salt electrolytes. In contrast, the TFSI $^{-}$ anions dominate the overall transport for all compositions except for the case in which the pure IL is employed, where $t^{\mathrm{NMR}}\left(\mathrm{Pyr}_{14}{ }^{+}\right)$shows the largest contribution. Note that the transport number of $\mathrm{TFSI}^{-}$only marginally decreases with increasing amount of IL. When using $\mathrm{LiPF}_{6}$ as a conducting salt, the anionic transport contributions change due to the fact that two negatively charged species are present. Without Pyr $_{14}$ TFSI, the total molecular transport is facilitated to about $40 \%$ by $\mathrm{Li}^{+}$and to about $60 \%$ by $\mathrm{PF}_{6}{ }^{-}$. As before, when adding $\mathrm{Pyr}_{14}$ TFSI, the cationic transport becomes more and more dominated by $\mathrm{Pyr}_{14}{ }^{+}$ ions. At the same time, the initially large contribution of $\mathrm{PF}_{6}{ }^{-}$ decreases at the expense of the $\mathrm{TFSI}^{-}$contribution. Remarkably, $t^{\mathrm{NMR}}\left(\mathrm{PF}_{6}{ }^{-}\right)$becomes comparable to that of lithium in the limit 
of high IL concentrations, though $\mathrm{PF}_{6}{ }^{-}$shows the largest transport numbers for IL fractions smaller than $40 \mathrm{wt} \%$. Thus, for an IL fraction of $100 \mathrm{wt} \%$, the transport numbers are similar to those of an electrolyte containing LiTFSI as conducting salt. In the IL-free electrolyte, the lithium transport number is marginally smaller than for respective LiTFSI-containing electrolytes. In total, however, the overall trend of the lithium transport numbers is similar in both types of blends. It is evident that the addition of $\mathrm{Pyr}_{14}$ TFSI to the carbonate-based electrolyte leads to a decrease of the lithium transport number, which likely also impedes the overall performance of a battery cell relying on this type of electrolyte. Though one might intuitively expect that partly exchanging the ion species (such as the use of another conducting salt) relieves this problem due to an altered coordination of the lithium ions, our results indicate no such effect, at least for the systems under consideration. We note, however, that reasonable transport properties are still guaranteed for intermediate IL concentrations.

In contrast to the PFG NMR data, transference numbers can be extracted from MD simulations in a straightforward manner. To this purpose, the double sum in eqn (6) defining the total conductivity may be rewritten as:

$$
\begin{aligned}
\sigma= & \lim _{t \rightarrow \infty} \frac{e^{2}}{6 t V k_{\mathrm{B}} T}\left(\sum_{i=j}^{N}\left\langle z_{i}^{\alpha} z_{i}^{\alpha}\left[\Delta r_{i}(t) \Delta r_{i}(t)\right]\right\rangle\right. \\
& +\sum_{i \neq j}^{N}\left\langle z_{i}^{\alpha} z_{j}^{\alpha}\left[\Delta r_{i}(t) \Delta r_{j}(t)\right]\right\rangle \\
& \left.+\sum_{i \neq j, \alpha \neq \beta}^{N}\left\langle z_{i}^{\alpha} z_{j}^{\beta}\left[\Delta r_{i}(t) \Delta r_{j}(t)\right]\right\rangle\right)
\end{aligned}
$$

In this way, eqn (6) is separated into contributions arising from the self-diffusion of ions (first sum in eqn (10)), from crosscorrelations between ions of the same type (second sum in eqn (10), with the superscript labelling the ion type), and cross correlations among ions of different types (third sum in eqn (10)). For example, in case of blends containing LiTFSI, we have (by additionally introducing a more compact notation):

$$
\begin{gathered}
\sigma=\sigma_{\mathrm{Li}-\mathrm{Li}}^{\text {self }}+\sigma_{\mathrm{Pyr}-\mathrm{Pyr}}^{\text {self }}+\sigma_{\mathrm{TFSI}-\mathrm{TFSI}}^{\text {self }}+\sigma_{\mathrm{Li}-\mathrm{Li}}^{\text {cross }}+\sigma_{\mathrm{Pyr}-\mathrm{Pyr}}^{\text {cross }}+\sigma_{\mathrm{TFSI}-\mathrm{TFSI}}^{\text {cross }} \\
+\sigma_{\mathrm{Li}-\mathrm{Pyr}}^{\text {cross }}+\sigma_{\mathrm{Li}-\mathrm{TFSI}}^{\text {cross }}+\sigma_{\mathrm{Pyr}-\mathrm{TFSI}}^{\text {cross }}
\end{gathered}
$$

where the first three terms correspond to contributions arising from self-diffusion coefficients (PFG NMR), the next three contributions from cross correlations of different ions of the same type, and the last three terms define individual contributions of cross correlations between ions of different types.

While obtaining these individual contributions to conductivity from simulations requires substantial statistics, for the concentration range between 0 and $50 \mathrm{wt} \%$ of IL, we are confident that our simulations are sufficiently long to provide reliable estimates of these correlations, as verified by analyzing the dependence of the conductivity contributions on the observation time (not shown). In Fig. 5, we plotted the normalized (by total conductivity) contributions of cross correlations to the achievable ionic conductivity. Note that for systems with LiTFSI, there are total six of such correlations, while in $\mathrm{LiPF}_{6}$ systems there are ten.
Careful examination of Fig. 5 provides several interesting observations. Most cross-correlations have negative contributions to the conductivity, but some can be as high as $50 \%$ of the conductivity magnitude (e.g., TFSI-TFSI correlation in systems with LiTFSI salt and substantial content of IL). However, there are few correlations, which have a positive contribution such as the Li-TFSI correlation that actually contributes positively in systems with IL concentrations exceeding $10 \mathrm{wt} \%$ in both blends. In both systems, there is a strong contribution from correlations of ions of the same type. Such contributions show substantial composition dependence but often with opposite trends, which results in relatively constant combined contribution from these terms. For example, in the LiTFSI system, the $\mathrm{Pyr}_{14}-\mathrm{Pyr}_{14}$ correlation is increasing with increasing IL concentration while $\mathrm{Li}-\mathrm{Li}$ correlation is reduced. Cross-correlations of different type ions show a relatively concentration independent behavior, with exception of several Li-anion and Li-cation correlations, which seem to show a stronger composition dependence.

Having explicitly resolved the contributions from each type of ion-ion correlation to conductivity allows to directly estimate the transference numbers from simulation data. Specifically of interest here is the $\mathrm{Li}^{+}$transference number, which for electrolytes with LiTFSI salt can be defined as:

$$
t_{\mathrm{Li}}^{\sigma}=\left(\sigma_{\mathrm{Li}-\mathrm{Li}}^{\text {self }}+\sigma_{\mathrm{Li}-\mathrm{Li}}^{\text {cross }}+0.5 \sigma_{\mathrm{Li}-\mathrm{Pyr}}^{\text {cross }}+0.5 \sigma_{\mathrm{Li}-\mathrm{TFSI}}^{\text {cross }}\right) / \sigma
$$

For both blends we have calculated the $\mathrm{Li}^{+}$transference numbers and compared them with the $\mathrm{Li}^{+}$transport numbers obtained using self-diffusion coefficients as shown in Table 2. Despite rather substantial contributions from ion correlated motion to the ionic conductivity, the obtained $\mathrm{Li}^{+}$transference numbers are very similar to transport numbers in these systems, indicating that $\mathrm{Li}^{+}$ion diffusion and charge transport due to $\mathrm{Li}^{+}$are affected the same way by the correlated motion of ions. Nonetheless, it is important to emphasize that ion correlations do affect the overall transport, as reflected by the ratio of $\sigma_{\text {Impedance }}$ and $\sigma_{\mathrm{NMR}}$.

\section{Viscosity-related transport}

We also elucidated the role of the electrolyte viscosity for the net transport. To this end, we plotted the experimentally measured ionic conductivity as a function of the inverse viscosity to probe if both quantities are related by a linear dependence (Fig. 6). Also, we normalized the ionic conductivities in Fig. 6 by the total concentration of charge carriers (that is the sum of concentrations of LiTFSI/LiPF 6 and Pyr $_{14}$ TFSI). We found that all curves of the individual electrolytes almost fall onto a single master curve for both conducting salts (note that the deviating data point of pure IL/LiTFSI at the lowest temperature was measured at the onset of solidification). For both types of blends, however, there are slight variations of the conductivity with composition (more pronounced for $\mathrm{LiPF}_{6}$ ), likely resulting from residual impact of ionic correlations. The data points apparently deviate slightly from the linear trend in Fig. 6 at higher temperatures, which partially could be also due to contributions from convection within the electrolytes. Though correlations between the different ion species are clearly present (Fig. 5), their net effect is nearly independent of the composition of the considered electrolyte. Rather, the overall conductivity is largely 

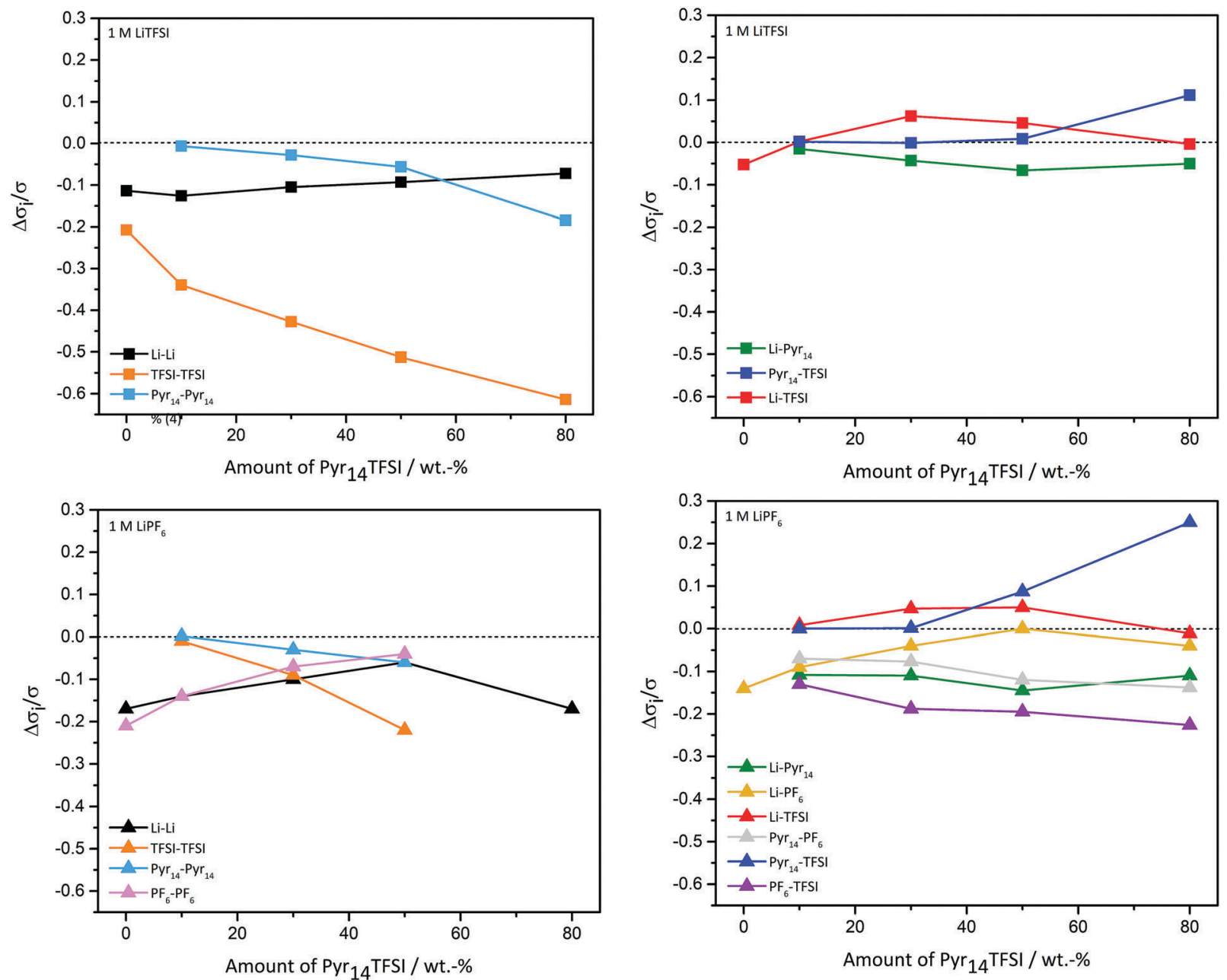

Fig. 5 Relative contributions of different ionic correlations defined by eqn (10) and (11) to the net conductivity of Pyr 14 TFSI/EC/DMC blends with 1 M LiTFSI (top) and $1 \mathrm{M} \mathrm{LiPF}_{6}$ (bottom) as conducting salts: correlations between distinct ions of the same type (left) and correlations between different ion types in the electrolyte (right). The lines are guides to the eye.

Table $2 \mathrm{Li}$ transport and transference numbers as obtained from MD simulations at $25^{\circ} \mathrm{C}$. All data is accurate within \pm 0.02

\begin{tabular}{|c|c|c|c|c|}
\hline \multirow{2}{*}{$\begin{array}{l}\text { Amount of } \\
\text { Pyr }_{14} \text { TFSI/wt } \%\end{array}$} & \multicolumn{2}{|l|}{$\mathrm{LiPF}_{6}$} & \multicolumn{2}{|l|}{ LiTFSI } \\
\hline & Transference & Transport & Transference & Transport \\
\hline 0 & 0.43 & 0.43 & 0.49 & 0.47 \\
\hline 10 & 0.37 & 0.35 & 0.42 & 0.37 \\
\hline 30 & 0.26 & 0.25 & 0.27 & 0.26 \\
\hline 50 & 0.21 & 0.19 & 0.24 & 0.20 \\
\hline 80 & 0.12 & 0.14 & 0.11 & 0.13 \\
\hline
\end{tabular}

determined by the viscosity of the electrolyte, apart from its trivial dependence on the total ion concentration. This is also consistent with the fact that the pseudo-activation energies from the VTF fits to the conductivity data are somewhat smaller than the respective values extracted from the viscosity data, and with the approximately constant degree of dissociation (see above). Our data clearly indicate that for a future development of novel electrolytes, the degree of ion dissociation (as determined by a significant coordination of solvent molecules to at least one of the major charge carriers) on the one hand and the viscosity on the other hand should not be optimized independently. Typically, the degree of ion dissociation is interpreted in terms of ion pairs or aggregates, leading to dynamical correlations between the motion of different ions (in particular cations and anions) that decrease the overall conductivity. It should be noted, however, that despite the fact that the coordination structurally changes from an organic carbonate-based lithium coordination to an anion-dominated coordination, the overall effect of dynamical ion correlations (as expressed by the displacement vectors of the ions devoid of any structural information, see eqn (6)) is nearly independent of the actual IL fraction, though the individual contributions depend on the electrolyte composition (Fig. 5). The conductivity is essentially determined by the viscosity of the electrolyte. Despite that this key parameter has been in the focus of electrolyte optimization for a long time, it nevertheless remains challenging to derive precise microscopic information from experimental viscosity data. This is essentially due to the fact that for the viscosity not only structural or dynamical interactions between ions are relevant, but also coordination between ions and uncharged solvent molecules. 

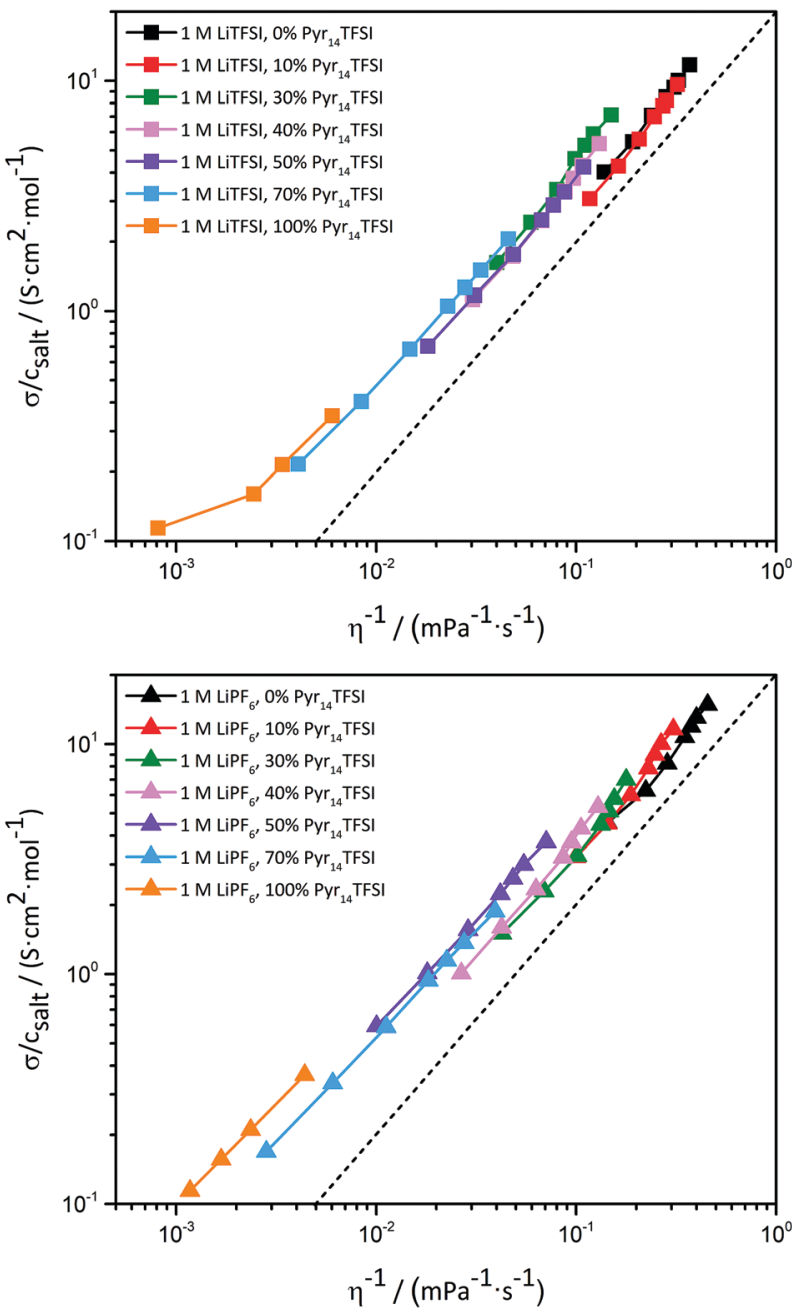

Fig. 6 Concentration-normalized conductivities of $\mathrm{Pyr}_{14}$ TFSI/EC/DMC blends with $1 \mathrm{M} \mathrm{LiTFSI}$ (top) and $1 \mathrm{M} \mathrm{LiPF}_{6}$ (bottom) from impedance measurements as function of inverse electrolyte viscosity. The conductivity values were normalized by the total salt concentration (i.e., the sum of the concentrations of both LiTFSI/LiPF 6 and $\mathrm{Pyr}_{14}$ TFSI). The dashed lines $(y=20 x)$ and solid lines are guides to the eye.

Therefore, explicitly relating the ion coordination structure to particular viscosities on the basis of MD simulations via the pressure tensor (thus generalizing the analysis of dynamical ion correlations in some sense) appears to be an interesting strategic way for future research.

\section{Conclusions}

Additives and co-solvents are known to improve the properties of electrolytes for lithium ion and lithium metal batteries. Mixing of ILs and organic carbonates offers distinctive advantages, ${ }^{86}$ where either carbonates are added to ILs or vice versa, including enhancement of electrochemical properties (e.g., cycle life or rate capacities). ${ }^{87}$ In other cases improved interfacial behavior of the electrolytes was observed, though the exact nature of interfacial interactions between different IL-based liquid/solid electrolytes and available electrode materials are not fully understood. ${ }^{88}$
In particular, we investigated the impact of the amount of $\mathrm{Pyr}_{14}$ TFSI in EC/DMC solvent blends containing $1 \mathrm{M}$ conducting salt (LiTFSI or $\mathrm{LiPF}_{6}$ ) on the actual transport and solvation properties of lithium ions as well as electrochemical properties combining experimental and molecular dynamics simulation data. We observed an excellent agreement of the simulation results with the experimental data, particularly diffusion and conductivity measurements by PFG NMR, impedance and Raman spectroscopy characterizing the lithium ion coordination. Key quantities derived from MD simulations include dynamical ion correlations among various ion species thereby unraveling which species tend to migrate cooperatively within the electrolyte (either by moving on average into the same or into opposite directions), in this way affecting the net charge transport that determines the achievable electrochemical performance of chemical storage devices. Remarkably, we identified that though the individual correlations between the different ion types show a clear concentration dependence, their net effect is almost constant throughout the entire concentration range. This is also reflected by the fact that the conductivities based on impedance measurements are by $\sim 40 \%$ lower than the respective ideal conductivities (based on diffusion measurements) for all considered Pyr $_{14}$ TFSI concentrations, though the absolute ion transport decreases by roughly one order of magnitude when going from carbonate solvent-based to pure ionic liquid-based electrolytes. The analysis of the MD data allowed us to compute transference numbers for the lithium ions, which are inaccessible by impedance spectroscopy and PFG NMR diffusion measurements alone (as opposed to transport numbers that are based on self-diffusion coefficients). In agreement with our previous observations, however, we established that both transference and transport numbers are approximately identical due to the almost constant net effect of dynamical ion correlations in our particular case. This is even more remarkable in view of the fact we monitored a crossover from a carbonate-based lithium ion coordination to a TFSI-based coordination. Notably, rather than dynamical ion correlations, we found that absolute values of the ionic conductivity are essentially determined by both the overall viscosity of the electrolyte, as well as the total ion concentration, hence illustrating that the achievable transport properties are only marginally affected by the explicit choice of the conducting salt (LiTFSI or LiPF $_{6}$ ).

Considering practical applications, we conclude that ionic liquid/carbonate solvent electrolyte blends with a $\mathrm{Pyr}_{14} \mathrm{TFSI}$ fraction of $\sim 10 \mathrm{wt} \%$ appear to be most promising, as in this case, the conductivity values are comparable to those of industrially relevant carbonate-based electrolytes and the lithium ion transference number is still $\sim 0.4$ as typically observed for standard electrolytes. $^{89}$ At the same time, the thermal and electrochemical stabilities of such electrolytes containing $\sim 10 \mathrm{wt} \%$ Pyr $_{14}$ TFSI are noticeably improved. From a theoretical perspective, our results strongly suggest to focus on the microscopic interplay between structural electrolyte properties such as preferential ion coordination or the formation of specific (charged or uncharged) aggregates on the one hand and the overall viscosity on the other hand rather than on ion correlations alone. In this respect, a separation of electrolyte viscosities into individual contributions 
from specific molecular species in MD simulations appears to be an interesting and versatile strategic route. Despite compositional changes of individual contributions, the ionic conductivity essentially depends on the total viscosity of considered electrolytes, thereby offering a rather straightforward criterion for future development of novel electrolytes. A crucial step includes high performance electrolyte components such as solvents, conducting salts and additives, facilitating an optimum viscosity as well as high thermal and electrochemical stability, and longterm cycling stability over a wide range of potentials $(0-5.5 \mathrm{~V} v$ s. $\left.\mathrm{Li} / \mathrm{Li}^{+}\right)$. Also, in view of battery application, novel electrolytes should enable reproducible formation of stable, effective electrolyte/electrode interfaces and interphases, as extensively discussed elsewhere. ${ }^{25,40}$

\section{Conflicts of interest}

There are no conflicts to declare.

\section{Acknowledgements}

Special thanks are offered to Mariano Grünebaum, who provided the micro-electrode liquid measurement cells, and to Anna Gerlitz who performed the impedance measurements. We also thank Verena Naber and Debbie Berghus who performed the DSC and TGA measurements. D. B., M. E. and J. B. H. gratefully acknowledge the support from the project sponsored by the Army Research Laboratory under Cooperative Agreement Number W911NF-12-2-0023. The views and conclusions contained in this document are those of the authors and should not be interpreted as representing the official policies, either expressed or implied, of ARL or the U.S. Government. The U.S. Government is authorized to reproduce and distribute reprints for Government purposes notwithstanding any copyright notation herein. We also would like to acknowledge the Center of High Performance Computing at the University of Utah for the generous allocation of computing resources and technical support.

\section{References}

1 T. Placke, R. Kloepsch, S. Dühnen and M. Winter, J. Solid State Electrochem., 2017, 21, 1939-1964.

2 S. Hess, M. Wohlfahrt-Mehrens and M. Wachtler, J. Electrochem. Soc., 2015, 162, A3084-A3097.

3 Y. Wang, K. Zaghib, A. Guerfi, F. F. C. Bazito, R. M. Torresi and J. R. Dahn, Electrochim. Acta, 2007, 52, 6346-6352.

4 P. Meister, H. Jia, J. Li, R. Kloepsch, M. Winter and T. Placke, Chem. Mater., 2016, 28, 7203-7217.

5 R. Wagner, N. Preschitschek, S. Passerini, J. Leker and M. Winter, J. Appl. Electrochem., 2013, 43, 481-496.

6 A. Khan and C. Zhao, ACS Sustainable Chem. Eng., 2015, 4, 506-513.

7 L. Cecchetto, M. Salomon, B. Scrosati and F. Croce, J. Power Sources, 2012, 213, 233-238.
8 M. Marcinek, J. Syzdek, M. Marczewski, M. Piszcz, L. Niedzicki, M. Kalita, A. Plewa-Marczewska, A. Bitner, P. Wieczorek, T. Trzeciak, M. Kasprzyk, P. Łężak, Z. Zukowska, A. Zalewska and W. Wieczorek, Solid State Ionics, 2015, 276, 107-126.

9 V. Aravindan, J. Gnanaraj, S. Madhavi and H. K. Liu, Chem. Eur. J., 2011, 17, 14326-14346.

10 R. W. Schmitz, P. Murmann, R. Schmitz, R. Müller, L. Krämer, J. Kasnatscheew, P. Isken, P. Niehoff, S. Nowak, G.-V. Röschenthaler, N. Ignatiev, P. Sartori, S. Passerini, M. Kunze, A. Lex-Balducci, C. Schreiner, I. Cekic-Laskovic and M. Winter, Prog. Solid State Chem., 2014, 42, 65-84.

11 K. Xu, Chem. Rev., 2014, 114, 11503-11618.

12 S. Nowak and M. Winter, J. Electrochem. Soc., 2015, 162, A2500-A2508.

13 J. Mindemark, M. J. Lacey, T. Bowden and D. Brandell, Prog. Polym. Sci., 2018, 81, 114-143.

14 Z. Xue, D. He and X. Xie, J. Mater. Chem. A, 2015, 3, 19218-19253. 15 H. Zhang, C. Li, M. Piszcz, E. Coya, T. Rojo, L. M. RodriguezMartinez, M. Armand and Z. Zhou, Chem. Soc. Rev., 2017, 46, 797-815.

16 A. Mauger, M. Armand, C. M. Julien and K. Zaghib, J. Power Sources, 2017, 353, 333-342.

17 A. Balducci, Top. Curr. Chem., 2017, 375, 20.

18 J. Kasnatscheew, R. Wagner, M. Winter and I. Cekic-Laskovic, Top. Curr. Chem., 2018, 376, DOI: 10.1007/s41061-018-0196-1.

19 S. Kazemiabnavi, Z. Zhang, K. Thornton and S. Banerjee, J. Phys. Chem. B, 2016, 120, 5691-5702.

20 F. Castiglione, E. Ragg, A. Mele, G. Battista Appetecchi, M. Montanino and S. Passerini, J. Phys. Chem. Lett., 2011, 2, 153-157.

21 M. Kunze, S. Jeong, E. Paillard, M. Winter and S. Passerini, J. Phys. Chem. C, 2010, 114, 12364-12369.

22 A. Deshpande, L. Kariyawasam, P. Dutta and S. Banerjee, J. Phys. Chem. C, 2013, 117, 25343-25351.

23 Z. Li, O. Borodin, G. D. Smith and D. Bedrov, J. Phys. Chem. $B, 2015,119$, 3085-3096.

24 G. H. Wrodnigg, J. O. Besenhard and M. Winter, J. Power Sources, 2001, 97-98, 592-594.

25 I. Cekic-Laskovic, N. von Aspern, L. Imholt, S. Kaymaksiz, K. Oldiges, B. R. Rad and M. Winter, Top. Curr. Chem., 2017, $375,37$.

26 S. Higashiya, T. S. Devarajan, M. V. Rane-Fondacaro and P. Haldar, ECS Trans., 2012, 41, 103-109.

27 V. Ruiz, T. Huynh, S. R. Sivakkumar and A. G. Pandolfo, RSC Adv., 2012, 2, 5591.

28 R. S. Kühnel, N. Böckenfeld, S. Passerini, M. Winter and A. Balducci, Electrochim. Acta, 2011, 56, 4092-4099.

29 A. Guerfi, M. Dontigny, P. Charest, M. Petitclerc, M. Lagacé, A. Vijh and K. Zaghib, J. Power Sources, 2010, 195, 845-852.

30 R.-S. Kühnel, M. Lübke, M. Winter, S. Passerini and A. Balducci, J. Power Sources, 2012, 214, 178-184.

31 B. Garcia and M. Armand, J. Power Sources, 2004, 132, 206-208.

32 L. Lombardo, S. Brutti, M. A. Navarra, S. Panero and P. Reale, J. Power Sources, 2013, 227, 8-14.

33 G. H. Lane, A. S. Best, D. R. MacFarlane, M. Forsyth, P. M. Bayley and A. F. Hollenkamp, Electrochim. Acta, 2010, 55, 8947-8952. 
34 T. M. W. J. Bandara and B.-E. Mellander, Evaluation of Mobility, Diffusion Coefficient and Density of Charge Carriers in Ionic Liquids and Novel Electrolytes Based on a New Model for Dielectric Response, InTech, 2011.

35 T.-Y. Wu, L. Hao, C.-W. Kuo, Y.-C. Lin, S.-G. Su, P.-L. Kuo and I.-W. Sun, Int. J. Electrochem. Sci., 2012, 7, 2047-2064.

36 S. Jeremias, G. A. Giffin, A. Moretti, S. Jeong and S. Passerini, J. Phys. Chem. C, 2014, 118, 28361-28368.

37 T. Frömling, M. Kunze, M. Schönhoff, J. Sundermeyer and B. Roling, J. Phys. Chem. B, 2008, 112, 12985-12990.

38 C. Arbizzani, G. Gabrielli and M. Mastragostino, J. Power Sources, 2011, 196, 4801-4805.

39 L. Aguilera, J. Scheers and A. Matic, Phys. Chem. Chem. Phys., 2016, 18, 25458-25464.

40 M. Amereller, T. Schedlbauer, D. Moosbauer, C. Schreiner, C. Stock, F. Wudy, S. Zugmann, H. Hammer, A. Maurer, R. M. Gschwind, H. D. Wiemhöfer, M. Winter and H. J. Gores, Prog. Solid State Chem., 2014, 42, 39-56.

41 T. Böttcher, B. Duda, N. Kalinovich, O. Kazakova, M. Ponomarenko, K. Vlasov, M. Winter and G. V. Röschenthaler, Prog. Solid State Chem., 2014, 42, 202-217.

42 M. Dahbi, F. Ghamouss, F. Tran-Van, D. Lemordant and M. Anouti, J. Power Sources, 2011, 196, 9743-9750.

43 L. J. Krause, W. Lamanna, J. Summerfield, M. Engle, G. Korba, R. Loch and R. Atanasoski, J. Power Sources, 1997, 68, 320-325.

44 H. Yang, K. Kwon, T. M. Devine and J. W. Evans, J. Electrochem. Soc., 2000, 147, 4399-4407.

45 E. Krämer, S. Passerini and M. Winter, ECS Electrochem. Lett., 2012, 1, C9-C11.

46 E. Krämer, T. Schedlbauer, B. Hoffmann, L. Terborg, S. Nowak, H. J. Gores, S. Passerini and M. Winter, J. Electrochem. Soc., 2013, 160, A356-A360.

47 M. Grünebaum, M. M. Hiller and H.-D. Wiemhöfer, GER Pat., DE102013004204A1, 2014.

48 M. Grünebaum, M. M. Hiller and H.-D. Wiemhöfer, GER Pat., WO2014139494A1, 2014.

49 O. Borodin and G. D. Smith, J. Phys. Chem. B, 2006, 110, 6279-6292.

50 O. Borodin and G. D. Smith, J. Phys. Chem. B, 2006, 110, 6293-6299.

51 O. Borodin, J. Phys. Chem. B, 2009, 113, 11463-11478.

52 G. J. Martyna, M. L. Klein and M. Tuckerman, J. Chem. Phys., 1992, 97, 2635-2643.

53 B. J. Palmer, J. Comput. Phys., 1993, 104, 470-472.

54 J.-P. Ryckaert, G. Ciccotti and H. J. Berendsen, J. Comput. Phys., 1977, 23, 327-341.

55 G. J. Martyna, D. J. Tobias and M. L. Klein, J. Chem. Phys., 1994, 101, 4177-4189.

56 G. J. Martyna, M. E. Tuckerman, D. J. Tobias and M. L. Klein, Mol. Phys., 1996, 87, 1117-1157.

57 D. Diddens, E. Paillard and A. Heuer, J. Electrochem. Soc., 2017, 164, E3225-E3231.

58 D. Diddens and A. Heuer, J. Phys. Chem. B, 2014, 118, 1113-1125.

59 D. Diddens and A. Heuer, ACS Macro Lett., 2013, 2, 322-326.

60 D. Diddens, A. Heuer and O. Borodin, Macromolecules, 2010, 43, 2028-2036.
61 G. Brunklaus, S. Schauff, D. Markova, M. Klapper, K. Müllen and H.-W. Spiess, J. Phys. Chem. B, 2009, 113, 6674-6681.

62 V. Lesch, S. Jeremias, A. Moretti, S. Passerini, A. Heuer and O. Borodin, J. Phys. Chem. B, 2014, 118, 7367-7375.

63 H. S. Shiau, W. Liu, R. H. Colby and M. J. Janik, J. Chem. Phys., 2013, 139, 204905.

64 Y. Shen, G. H. Deng, C. Ge, Y. Tian, G. Wu, X. Yang, J. Zheng and K. Yuan, Phys. Chem. Chem. Phys., 2016, 18, 14867-14873.

65 K. Kimura, J. Motomatsu and Y. Tominaga, J. Phys. Chem. C, 2016, 120, 12385-12391.

66 R.-S. Kühnel and A. Balducci, J. Phys. Chem. C, 2014, 118, 5742-5748.

67 O. Borodin, M. Olguin, P. Ganesh, P. R. Kent, J. L. Allen and W. A. Henderson, Phys. Chem. Chem. Phys., 2016, 18, 164-175.

68 D. M. Seo, S. Reininger, M. Kutcher, K. Redmond, W. B. Euler and B. L. Lucht, J. Phys. Chem. C, 2015, 119, 14038-14046.

69 M. Morita, Y. Asai, N. Yoshimoto and M. Ishikawa, J. Chem. Soc., Faraday Trans., 1998, 94, 3451-3456.

70 M. Castriota, T. Caruso, R. G. Agostino, E. Cazzanelli, W. A. Henderson and S. Passerini, J. Phys. Chem. A, 2005, 109, 92-96.

71 D. Brouillette, D. E. Irish, N. J. Taylor, G. r. Perron, M. Odziemkowski and J. E. Desnoyers, Phys. Chem. Chem. Phys., 2002, 4, 6063-6071.

72 S. Menne, T. Vogl and A. Balducci, Phys. Chem. Chem. Phys., 2014, 16, 5485-5489.

73 U. Olsher, Chem. Rev., 1991, 91, 137-164.

74 L. J. Hardwick, M. Holzapfel, A. Wokaun and P. Novák, J. Raman Spectrosc., 2007, 38, 110-112.

75 D. M. Seo, O. Borodin, S. D. Han, P. D. Boyle and W. A. Henderson, J. Electrochem. Soc., 2012, 159, A1489-A1500.

76 B. Dünweg and K. Kremer, J. Chem. Phys., 1993, 99, 6983-6997.

77 I.-C. Yeh and G. Hummer, J. Phys. Chem. B, 2004, 108, 15873-15879.

78 S. Gabl, C. Schröder and O. Steinhauser, J. Chem. Phys., 2012, 137, 094501.

79 W. M. Haynes, CRC Handbook of Chemistry and Physics, Taylor \& Francis, Boca Raton, 91st edn, 2010.

80 C. A. Angell, Electrochim. Acta, 2017, 250, 368-375.

81 G. Murch, Solid State Ionics, 1982, 7, 177-198.

82 S. Zugmann, M. Fleischmann, M. Amereller, R. M. Gschwind, M. Winter and H. J. Gores, J. Chem. Eng. Data, 2011, 56, 4786-4789.

83 A. K. Sethurajan, S. A. Krachkovskiy, I. C. Halalay, G. R. Goward and B. Protas, J. Phys. Chem. B, 2015, 119, 12238-12248.

84 F. Wohde, M. Balabajew and B. Roling, J. Electrochem. Soc., 2016, 163, A714-A721.

85 P. G. Bruce and C. A. Vincent, J. Electroanal. Chem., 1987, 225, 1-17.

86 A. Eftekhari, Y. Liu and P. Chen, J. Power Sources, 2016, 334, 221-239.

87 Z. Wang, Y. Cai, Z. Wang, S. Chen, X. Lu and S. Zhang, J. Solid State Electrochem., 2013, 17, 2839-2848.

88 Q. Yang, Z. Zhang, X. G. Sun, Y. S. Hu, H. Xing and S. Dai, Chem. Soc. Rev., 2018, 47, 2020-2064.

89 S. Zugmann, M. Fleischmann, M. Amereller, R. M. Gschwind, H. D. Wiemhöfer and H. J. Gores, Electrochim. Acta, 2011, 56, 3926-3933. 